See discussions, stats, and author profiles for this publication at: https://www.researchgate.net/publication/258836432

\title{
A Comprehensive Centralized Approach for Voltage Constraints Management in Active Distribution Grid
}

Article in IEEE Transactions on Power Systems · March 2014

DOI: 10.1109/TPWRS.2013.2287897

CITATIONS

93

3 authors:

Florin Capitanescu

Luxembourg Institute of Science and Technology (LIST)

87 PUBLICATIONS 2,336 CITATIONS

SEE PROFILE

Esther Romero-Ramos

Universidad de Sevilla

45 PUBLICATIONS 820 CITATIONS

SEE PROFILE

Some of the authors of this publication are also working on these related projects:

Project $\quad$ Autonomous supply with thermal and electrical energy for eco-cities View project

Project $\quad$ gENESiS View project
READS

267

Ilya Bilibin

University of Luxembourg

5 PUBLICATIONS 118 CITATIONS

SEE PROFILE 


\title{
A Comprehensive Centralized Approach for Voltage Constraints Management in Active Distribution Grid
}

\author{
Florin Capitanescu, Ilya Bilibin, and Esther Romero Ramos
}

\begin{abstract}
This paper deals with the management of voltage constraints in active distribution systems that host a significant amount of distributed generation (DG) units. To this end we propose a centralized optimization approach which aims at minimizing the amount of MW curtailment of non-firm DG to remove voltage constraints. The salient feature of this approach is that it comprehensively and properly models the full variety of possible control means (i.e. DG active/reactive power including DG shut-down, on load tap changing transformer ratio, shunt capacitor, and remotely controlled switches or breakers), most of which having a discrete behavior. We develop and compare the performances of two optimization models on a snapshot basis for various distribution systems up to 1089 buses. In particular we show that the use of remotely controlled switches so as to transfer DG between feeders in case of voltage constraints may lead to significant reduction of the DG curtailment.
\end{abstract}

Index Terms-active distribution system, distributed generation, network switching, optimal power flow, smart grid, voltage control, Volt/VAR control.

\section{LIST OF ACRONYMS}

This section gathers the main acronyms used through the paper.

$\begin{array}{ll}\text { DG } & \text { distributed generation. } \\ \text { DS } & \text { distribution system. } \\ \text { DSO } & \text { distribution system operator. } \\ \text { LTC } & \text { on load tap changing transformer. } \\ \text { MILP } & \text { mixed integer linear programming. } \\ \text { MINLP } & \text { mixed integer nonlinear programming. } \\ \text { MINLP-RC } & \text { mixed integer nonlinear programming with } \\ & \text { voltages expressed in rectangular coordinates. } \\ \text { MIQC } & \text { mixed integer quadratically constrained. } \\ \text { OPF } & \text { optimal power flow. } \\ \text { RCS } & \text { remotely controlled switches. } \\ \text { VC } & \text { voltage control. } \\ \text { VCM } & \text { voltage constraints management. }\end{array}$

\section{INTRODUCTION}

The real-time control of voltages in the traditionally passive distribution systems (DSs) has been usually performed by the classical Volt/VAR control [1], [2]. The latter relies on local automatic discrete voltage controllers such as the on load tap changing (LTC) and shunt capacitors switching and aims at maintaining voltages on the DS between their statutory limits. Nowadays, in order to meet the more stringent environmental

F. Capitanescu and I. Bilibin are with the Interdisciplinary Centre for Security, Reliability and Trust (SnT), University of Luxembourg, Luxembourg (e-mail: florin.capitanescu@uni.lu; ilya.bilibin@uni.lu). E. Romero Ramos is with the Department of Electrical Engineering, University of Sevilla, Sevilla (e-mail: eromero@us.es). constraints, many DSs host increasing amounts of distributed generation (DG), which, beyond some penetration limit, may lead to various operational problems such as thermal overload, protection malfunction, and voltage rise among others [3].

In the context of active ${ }^{1} D S$ paradigm voltage problems cannot be properly managed by classical Volt/VAR control means and hence the voltage control (VC) strategy needs to be redefined [4].

To this end various $\mathrm{VC}$ and/or voltage constraints management (VCM) schemes with different trade-offs between the investments cost (e.g. in additional two-ways communication, control and metering devices) and potential benefits have been proposed in the context of active/smart grids [5]-[21]. These schemes fall into two main classes: centralized [5][11], [13], [20] and distributed [13]-[19], [21]. The approaches from the former class rely on: optimal power flow (OPF) techniques applied to a snapshot [5], [9], [11]-[13] or timeseries [6], MILP OPF approximation [8], or model predictive control [10]. The decentralized approaches are mostly based on voltage sensitivity with respect to $\mathrm{DG}$ active and/or reactive injections which are embedded into an optimization-based strategy by: local DG reactive power or power factor control [13], [14], local DG units active/reactive power control [15], local LTC voltage setpoint control [16], multi agent systems [17]-[19], adaptive control [20], or hybrid schemes combining local and remote control [21].

These approaches can be further classified according to whether they are proven using static (snapshot) analysis [5], [7]-[9], [11]-[13], [17], [20] or simulated real-time environment and/or time-series [6], [10], [14]-[16], [18], [19], [21].

We notice that most previous works generally address the VC and VCM problems by considering only a subset among the available control means (i.e. mostly adjustments of LTC voltage set-point and DG active/reactive powers). Furthermore, except of very few works (e.g. [8], [9]), they disregard the discrete nature of most voltage control means, and except of Ref. [9], do not consider the option of using online remotely controlled switches $(\mathrm{RCS})^{2}$. However, [8], [9] do not consider the exact nonlinear problem but solely linear approximations. Furthermore, the MILP approach in Ref. [8]

\footnotetext{
${ }^{1}$ This is an intermediate step towards the concept of smart distribution grid and aims at increasing significantly the DG penetration level thanks to adequate real-time power flows management schemes.

${ }^{2}$ Modern distribution systems are equipped with remotely controlled switches. Furthermore, in their transition towards active DSs and smart grids, one expects that distribution systems will be equipped at strategic locations with an increasing number of remotely controlled switches and/or breakers (e.g. to maintain protection coordination in the presence of DG) that will greatly facilitate the fast grid control and improve reliability [9], [22].
} 
considers another objective function (e.g. loss minimization) and does not consider RCS or DG active power in the controls set, whereas the LP sensitivity-based approach in Ref. [9] does not include some discrete variables (e.g. LTC, shunts, and DG connection status) and deals with RCS by enumeration.

The main contribution of this work is to develop a comprehensive centralized VCM approach that considers the full complexity of the problem in terms of potential control variables (i.e. LTC ratio, shunt capacitor switching, remotely controlled switches/breakers, DG active/reactive power adjustments and DG units complete shut-down) and models properly the discrete control means. However, this leads to posing a mixed integer nonlinear programming (MINLP) problem, whereas solvers of this class of optimization problems are not able to comply with the stringent time requirements of the online control for a large number of discrete variables and/or large grids [23]-[25]. In this paper we show that, as most DSs are (or foreseeable will be) equipped with a relatively small number of discrete control means for VCM (e.g. RCS, LTCs, shunts), MINLP solvers generally provide acceptably fast solutions for properly formulated VCM problems. In this work we develop and compare performances of two optimization models for VCM in distribution grids:

- model MINLP-RC: a MINLP model which expresses complex voltages in Rectangular Coordinates (RC), so that to take advantage of less nonlinearity of constraints compared to the more common model which expresses voltages using polar coordinates [23].

- model MIQC: an equivalent mixed integer quadratically constrained (MIQC) model of the MINLP-RC model, which builds upon the model proposed in [27] for loss minimization by grid reconfiguration, and was adapted by the authors to overload management [28]. In this paper we further extend the latter model to the objective function and additional control means (e.g. LTC ratio and shunts) specific to VCM application and test it on larger DSs.

As other centralized VCM schemes [5]-[11], [13], [20] our approach relies in turn on the output of a state estimator. Recent research shown that estimated states of acceptable quality of can be obtained with a relatively small but properly deployed number of additional measurements [36], [37]. The state estimation may further take advantage from improved pseudo-measurements stemming from home smart meters, which installation is underway in several countries (e.g. in Europe) as a first step towards smart distribution grids.

The remaining of the paper is organized as follows. Sections II and III present the MINLP-RC and MIQC optimization models. Section IV provides numerical results with these models and section $\mathrm{V}$ concludes.

\section{THE MINLP-RC OPTIMIZATION MODEL}

\section{A. On the regulatory framework}

The two main regulatory frameworks of DG access to the grid rely on [29]: DG connection agreements [29], [30] and electricity markets [9], [31]. In the former framework, according to the DG connection agreement, one can distinguish between "firm"3 DG units and "non-firm"4 DG units [30]. In this framework thermal/voltage constraints are removed according to a given objective (e.g. minimizing either the MW curtailed or the curtailment cost [29]) or DG connection agreements (e.g. last-in, first-off [30]). In the second framework based on real-time electricity markets one generally looks for minimizing the Distribution System Operator (DSO) payments towards the owners of curtailed DG units [9], [29], [31], likewise in the transmission system.

One can conclude that most regulatory frameworks differ basically in two respects: the choice of non-firm DG units participating in curtailment and the optimization goal. Bearing this in mind we devise an optimization approach for voltage constraints management which is versatile enough so that to accommodate most regulatory frameworks (e.g. by properly choosing the objective function, some variables and some constraints), and hence be applicable in various contexts.

\section{B. The objective function}

Let $N, G, E, T, L, S, B$ denote the set of respectively: nodes, DG units, equivalent generators modeling the transmission system, LTC transformers, all lines, the subset of lines with remotely controlled switches/breakers, and shunt capacitor banks.

The goal of the optimization problem is to minimize, in some optimal manner that depends on the adopted regulatory framework, the amount of non-firm DG units MW curtailed to remove voltage violations. However, as distribution grid regulation and degree of automation differ from one country to another, in order to comprehensively and flexibly model various DSO options, we consider the following composite objective function that minimizes the weighted deviation of control means with respect to their current values:

$$
\begin{aligned}
\min & \sum_{i \in G} w_{P}\left(P_{g i}^{0}-P_{g i}\right)+\sum_{i \in G} w_{Q}\left|Q_{g i}^{0}-Q_{g i}\right|+ \\
& \sum_{i j \in T} w_{t}\left|t_{i j}-t_{i j}^{0}\right|+\sum_{i \in B} w_{p}\left|p_{s h i}-p_{s h i}^{0}\right|+ \\
& \sum_{i j \in S} w_{s}\left|s_{i j}-s_{i j}^{0}\right|+\sum_{i \in N} w_{V}\left(\delta_{i 1}+\delta_{i 2}\right)
\end{aligned}
$$

where: $P_{g i}^{0}, Q_{g i}^{0}, t_{i j}^{0}, p_{s h i}^{0}, s_{i j}^{0}$ denote the current value of respectively: the active/reactive power of DG unit $i$, the tap position of LTC $i j$, the discrete position number of shunt bank $i$, and the initial status connected/disconnected of switch/breaker $i j$, and can be obtained at the output of the state estimator [36], [37]. Furthermore:

- the term $w_{P}\left(P_{g i}^{0}-P_{g i}\right)$ is the major goal and, depending on the given context [29], looks for minimizing either the amount of MW curtailed on DG units or the cost of DSO payments towards the owners of curtailed DG;

\footnotetext{
${ }^{3}$ Generators that cannot be curtailed to remove grid constraints as they invested in grid reinforcement; these DG units are accommodated based on a worst-case scenario.

${ }^{4}$ Generators that accepted to be occasionally curtailed as grid congestion occurs because the lost revenue was deemed more advantageous economically than grid reinforcement option.
} 
- the term $w_{Q}\left|Q_{g i}^{0}-Q_{g i}\right|$ can account for cases where market arrangements exist to compensate the DG units that provide reactive power ancillary services [32];

- the terms $w_{t}\left|t_{i j}-t_{i j}^{0}\right|, w_{p}\left|p_{s h i}-p_{s h i}^{0}\right|$, and $w_{s}\left|s_{i j}-s_{i j}^{0}\right|$ aim to reduce the strain of switching operations on respectively: the LTC tap, the shunt capacitors, and remotely controlled switches/breakers;

- the term $w_{V}\left(\delta_{i 1}+\delta_{i 2}\right)$ penalizes the relaxation of voltage limits for infeasible problems and provides a trade-off between the original objective and voltage limit violations. Weight $w_{V}$ is chosen much larger than other weights to inhibit relaxing voltage limits for feasible problems.

Weights $w_{t}, w_{p}, w_{s}$ can be seen as maintenance costs for the switching actions on respectively: LTC tap, shunt banks and remotely controlled switches/breakers.

Notice that this objective function definition (1) is very versatile as a proper choice of the weights intervening in it allows establishing a priority list for the various control actions according to DSO needs and hence putting more emphasis on one particular term to the detriment of the others.

\section{The control variables and their corresponding constraints}

The control variables and their corresponding constraints are the following:

1) Connection status and active/reactive powers of DG:

$$
\begin{aligned}
& s_{g i} \in\{0,1\}, i \in G \\
& s_{g i} P_{g i}^{\min } \leq P_{g i} \leq s_{g i} P_{g i}^{0}, i \in G \\
& s_{g i} Q_{g i}^{\min } \leq Q_{g i} \leq s_{g i} Q_{g i}^{\max }, i \in G,
\end{aligned}
$$

where the binary variable $s_{g i}$ models the connection status of the generator $i\left(s_{g i}=1\right.$ if the generator is connected and $s_{g i}=0$ if it is shut down).

Note that constraints (4) assume that DG units can shift their reactive power so as to reduce the overall amount of active power curtailed but other typical DG reactive power control modes (e.g. constant power factor and in particular unitary power factor) can be taken into account straightforwardly.

For the sake of simplicity we model the DG units capability curves [33] by box active/reactive power constraints.

2) Active/reactive powers on the equivalent generators modeling the transmission system upstream the substation:

$$
\begin{aligned}
& P_{g i}^{\min } \leq P_{g i} \leq P_{g i}^{\max }, i \in E \\
& Q_{g i}^{\min } \leq Q_{g i} \leq Q_{g i}^{\max }, i \in E
\end{aligned}
$$

3) Connection status of remotely controlled switches:

$$
s_{i j} \in\{0,1\}, i j \in S
$$

4) Shunt banks integer positions:

$$
p_{s h i} \in s h_{i}, i \in B
$$

where $s h_{i}$ is the set of integer shunt banks positions.

The shunt susceptance can be expressed as:

$$
b_{s h i}=\left(p_{s h i}-1\right) \Delta b_{s h i},
$$

where the shunt susceptance step (assumed constant for simplicity) is given by $\Delta b_{s h i}=b_{s h i}^{\max } /\left(\left|s h_{i}\right|-1\right)$.

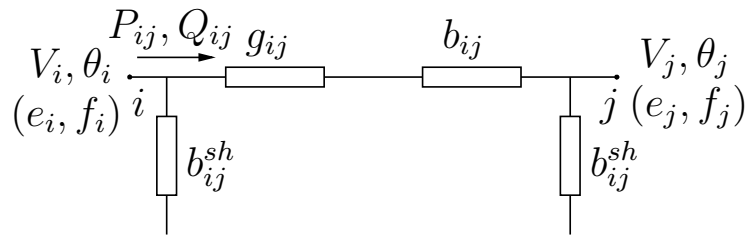

Fig. 1. Distribution line model

5) LTC transformer tap integer positions:

$$
t_{i j} \in t_{a p}, i j \in T
$$

where $t_{a p}$ ij is the set of integer values of the LTC tap.

The LTC transformer ratio (see Fig. 2) can be expressed as:

$$
r_{i j}=r_{i j}^{\min }+\left(t_{i j}-1\right) \Delta r_{i j},
$$

where $\Delta r_{i j}=\left(r_{i j}^{\max }-r_{i j}^{\min }\right) /\left(\left|\operatorname{tap}_{i j}\right|-1\right)$.

6) Relaxation variables on voltage limits constraints:

$$
\delta_{1 i}, \delta_{2 i} \geq 0, i \in N
$$

7) Other optimization variables: the real and imaginary part of bus complex voltage $e_{i}$ and $f_{i}$, as explained hereafter.

\section{Remaining constraints}

The problem is further subject to the following constraints:

1) Power flow equations: In our formulation we express complex voltages in rectangular coordinates:

$$
\underline{V}_{i}=e_{i}+j f_{i}, \quad i=1, \ldots, n,
$$

where $e_{i}$ and $f_{i}$ are its real and imaginary part respectively, the voltage magnitude being: $V_{i}=\sqrt{e_{i}^{2}+f_{i}^{2}}$.

For a classical line model shown in Fig. 1 the active/reactive power balance equations take on the form at bus $i \in N$ :

$$
\begin{aligned}
& P_{g i}-P_{c i}=\sum_{j \in N} s_{i j} P_{i j}= \\
& \sum_{j \in N} s_{i j}\left[g_{i j} V_{i}^{2}-\left(e_{i} e_{j}+f_{i} f_{j}\right) g_{i j}-\left(f_{i} e_{j}-e_{i} f_{j}\right) b_{i j}\right], \quad(13) \\
& Q_{g i}-Q_{c i}+V_{i}^{2} b_{s h i}=\sum_{j \in N} s_{i j} Q_{i j}= \\
& \sum_{j \in N} s_{i j}\left[\left(e_{i} e_{j}+f_{i} f_{j}\right) b_{i j}-\left(f_{i} e_{j}-e_{i} f_{j}\right) g_{i j}-\left(b_{i j}^{s h}+b_{i j}\right) V_{i}^{2}\right],
\end{aligned}
$$

where line switch status $s_{i j}$ properly models whether a line is switched on or off. Although the power flows through transformers (see Fig. 2) are not shown explicitly they can be written likewise.

2) Longitudinal branch current limits:

$$
\begin{aligned}
& I_{i j}^{2} \leq\left(I_{i j}^{\max }\right)^{2}, i j \in L \backslash S, \\
& I_{i j}^{2} \leq K_{I}\left(1-s_{i j}\right)+s_{i j}\left(I_{i j}^{\max }\right)^{2}, i j \in S,
\end{aligned}
$$

where the square of the longitudinal current is given by:

$$
I_{i j}^{2}=\left(g_{i j}^{2}+b_{i j}^{2}\right)\left[e_{i}^{2}+f_{i}^{2}+e_{j}^{2}+f_{j}^{2}-2\left(e_{i} e_{j}+f_{i} f_{j}\right)\right]
$$

and $K_{I}$ is a "bigM"-type constant properly chosen so that to relax constraints (16) for an open line. 
3) Relaxed voltage magnitude limits:

$$
V_{i \min }^{2}-\delta_{1 i} \leq e_{i}^{2}+f_{i}^{2} \leq V_{i \max }^{2}+\delta_{2 i}
$$

where $\delta_{1 i}$ and $\delta_{2 i}$ are positive relaxation terms (12).

4) Necessary radiality constraint:

$$
\sum_{i j \in S} s_{i j}=\sum_{i j \in S} s_{i j}^{0}
$$

which expresses the fact that the sum of statuses of lines with remote controlled switches must not change after reconfiguration. Because this constraint may be insufficient to ensure radiality in grids where there are some zero-injection nodes [27], we adopt a practical solution and replace each zeroinjection bus with a very small reactive power load (of value slightly above the power flow convergence tolerance), change which practically does not affect the result of the optimization.

5) Constraints limiting the number of switching actions:

$$
\begin{aligned}
& \sum_{i j \in S}\left|s_{i j}-s_{i j}^{0}\right| \leq \Delta S_{s w} \\
& \sum_{i j \in T}\left|t_{i j}-t_{i j}^{0}\right| \leq \Delta T_{s w} \\
& \sum_{i \in B}\left|p_{s h i}-p_{s h i}^{0}\right| \leq \Delta P_{s w}
\end{aligned}
$$

which models the DSO practical operational need that is not using more than a specified number of switching actions on discrete variables $\left(\Delta S_{s w}, \Delta T_{s w}\right.$, and $\left.\Delta P_{s w}\right)$ to remove voltage constraints in real-time. These constraints can also be extended so as to model DSO preference to limit the number of switching actions over a day.

\section{E. Remarks}

Note that, thanks to the model of voltages using rectangular coordinates, most constraints in the MINLP-RC optimization model (1)-(22) are at most quadratic whereas only power flow equations (13)-(14) are mildly nonlinear as they contain products between binary variables and quadratic variables, which reduces the inherent difficulty of a MINLP model.

Furthermore, unlike the (generally) off-line problem of network reconfiguration for loss minimization where all (manually and remotely controlled) switches are taken as decision variable, leading to a very large combinatorial space [27], [34], [35], in our VCM procedure this combinatorial space is drastically limited due to the small ratio between the number of remotely controlled switches/breakers and the number of all switches. Furthermore, the DSO needs to act on switches only whenever this action is very effective (e.g. by assigning an appropriate large cost to this control action in the objective (1)) may further implicitly limit this combinatorial space.

\section{THE MIQC OPTIMIZATION MODEL}

The model presented in this section builds on the model proposed in [27] for the minimization of losses problem by network reconfiguration. This model relies in turn on the power flow model for radial distribution systems proposed in [26], that we briefly describe in the next subsection.
A. Alternative power flow equations for radial distribution systems

For the line model shown in Fig. 1 the active/reactive power flows leaving the bus $i$ can be expressed as ${ }^{5}$ :

$$
\begin{aligned}
& P_{i j}=g_{i j} V_{i}^{2}-g_{i j} V_{i} V_{j} \cos \theta_{i j}-b_{i j} V_{i} V_{j} \sin \theta_{i j} \\
& Q_{i j}=-\left(b_{i j}+b_{i j}^{s h}\right) V_{i}^{2}+b_{i j} V_{i} V_{j} \cos \theta_{i j}-g_{i j} V_{i} V_{j} \sin \theta_{i j},
\end{aligned}
$$

where $\theta_{i j}=\theta_{i}-\theta_{j}$.

The model proposed in [26] consists, for a grid with $n+1$ nodes $^{6}$, in replacing the $2 n$ conventional nonlinear power flow equations with a set of $3 n$ equations ( $2 n$ linear and $n$ quadratic). This can be done by replacing the conventional complex voltage unknowns $\left(V_{i}, \theta_{i}\right)$ with two variables per branch $\left(W_{i j}\right.$ and $\left.T_{i j}\right)$ and one per bus $\left(U_{i}\right)$ as follows:

$$
\begin{aligned}
& U_{i}=V_{i}^{2} \\
& W_{i j}=V_{i} V_{j} \cos \theta_{i j} \\
& T_{i j}=V_{i} V_{j} \sin \theta_{i j},
\end{aligned}
$$

where $W_{i j}=W_{j i}$ and $T_{i j}=-T_{j i}$.

The new variables are related among each other as:

$$
U_{i} U_{j}=W_{i j}^{2}+T_{i j}^{2}
$$

Thanks to these new variables the branch active and reactive power flows take on linear expressions:

$$
\begin{aligned}
& P_{i j}=g_{i j} U_{i}-g_{i j} W_{i j}-b_{i j} T_{i j} \\
& Q_{i j}=-\left(b_{i j}+b_{i j}^{s h}\right) U_{i}+b_{i j} W_{i j}-g_{i j} T_{i j}
\end{aligned}
$$

Therefore the set of power flow equations proposed in [26] is made of $2 n$ linear equations:

$$
\begin{aligned}
& P_{g i}-P_{c i}=\sum_{j \in N} P_{i j}=\sum_{j \in N} g_{i j} U_{i}-g_{i j} W_{i j}-b_{i j} T_{i j} \\
& Q_{g i}-Q_{c i}+b_{s h i} U_{i}=\sum_{j \in N} Q_{i j}= \\
& \sum_{j \in N}-\left(b_{i j}+b_{i j}^{s h}\right) U_{i}+b_{i j} W_{i j}-g_{i j} T_{i j}
\end{aligned}
$$

together with the $n$ quadratic constraints (28).

At the solution of these power flow equations traditional voltage unknowns can be straightforwardly retrieved: $V_{i}$ from (25) and $\theta_{i}$ from (26) or (27) by exploring the grid tree downward from the slack bus substation.

\section{B. Other problem constraints}

The MIQC model inherits all control variables and equations from the model MINLP-RC (1)-(12) and (19)-(22), except of replacing optimization variables $e_{i}, f_{i}$ with the new variables $\left(U_{i}, W_{i j}\right.$, and $\left.T_{i j}\right)$ and expressing in a different way the status connected/disconnected of a line. Therefore constraints (13)-(18) are replaced by the following alternative constraints:

\footnotetext{
${ }^{5}$ We temporarily switch to a complex voltage representation by polar coordinates for the sake of explanation simplicity.

${ }^{6} \mathrm{We}$ assume here for simplicity a single substation but the model is generic to any number of substations.
} 


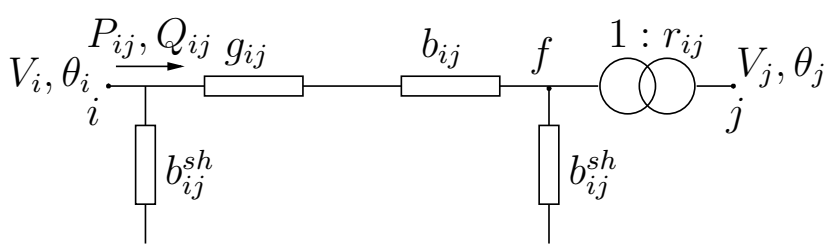

Fig. 2. LTC transformer model

1) Bus active/reactive power balance equations $(i \in N)$ :

$$
\begin{aligned}
& P_{g i}-P_{c i}=\sum_{j \in N} g_{i j} U_{i}-g_{i j} W_{i j}-b_{i j} T_{i j} \\
& Q_{g i}-Q_{c i}+Q_{s h i}=\sum_{j \in N}-\left(b_{i j}+b_{i j}^{s h}\right) U_{i}+b_{i j} W_{i j}-g_{i j} T_{i j}
\end{aligned}
$$

$Q_{s h i}=b_{s h i} U_{i}$

where the shunt banks susceptance $b_{s h i}$ is given by (9), and $Q_{s h i}$ is a substitution variable that aims maintaining equation (34) linear at the expense of introducing new quadratic equations (35).

2) Coupling constraints:

$$
U_{i} U_{j}=W_{i j}^{2}+T_{i j}^{2}, i j \in L \backslash S
$$

that complete the power flow equations for lines with fixed switches/breakers.

3) Additional quadratic constraints for LTC transformers: An LTC transformer can be modeled as shown in Fig. 2 by a $\pi$ model, likewise as for a line, in series with an ideal transformer with the ratio $r_{i j}$. As apparent powers at nodes $f$ and $j$ have opposite sign (i.e. $\underline{\mathbf{S}}_{f j}+\underline{\mathrm{S}}_{j f}=0$ ) the LTC transformer power flow equations (33)-(36) can be written for nodes $i$ and $f$, taking into account the relationship between voltages in the fictitious node $f$ and node $j$ that is $r_{i j} V_{f}=V_{j}$, and hence $r_{i j}^{2} U_{f}=U_{j}$. To avoid the nonlinearity of the latter relationship and keep the additional LTC constraints as quadratic we introduce a substitution variable $\tilde{r}_{i j}$ as follows:

$$
\begin{aligned}
& \tilde{r}_{i j} U_{f}=U_{j} \\
& \tilde{r}_{i j}=\left(r_{i j}\right)^{2}
\end{aligned}
$$

where $r_{i j}$ is given by (11).

4) Longitudinal branch current limits:

$$
I_{i j}^{2}=\left(g_{i j}^{2}+b_{i j}^{2}\right)\left(U_{i}+U_{j}-2 W_{i j}\right) \leq\left(I_{i j}^{\max }\right)^{2}, i j \in L \backslash S
$$

5) Constraints modeling the status of lines with remotely controlled switches/breakers (ij $\in S$ ): In order to model the network switching operation and consequently whether a branch is connected or disconnected we use the following set of constraints [27]:

$$
\begin{aligned}
& -s_{i j} P_{i j}^{\max } \leq P_{i j} \leq s_{i j} P_{i j}^{\max } \\
& -s_{i j} Q_{i j}^{\max } \leq Q_{i j} \leq s_{i j} Q_{i j}^{\max } \\
& -K_{I}\left(1-s_{i j}\right) \leq I_{i j}^{2} \leq K_{I}\left(1-s_{i j}\right)+s_{i j}\left(I_{i j}^{\max }\right)^{2} \\
& -K_{e}\left(1-s_{i j}\right) \leq U_{i} U_{j}-W_{i j}^{2}-T_{i j}^{2} \leq K_{e}\left(1-s_{i j}\right) \\
& -K_{W}\left(1-s_{i j}\right) \leq W_{i j}-W_{j i} \leq K_{W}\left(1-s_{i j}\right) \\
& -K_{T}\left(1-s_{i j}\right) \leq T_{i j}+T_{j i} \leq K_{T}\left(1-s_{i j}\right)
\end{aligned}
$$

TABLE I

DEFINITION OF CONTROL ACTIONS WEIGHS IN VARIOUS CASES

\begin{tabular}{|c|c|c|c|c|c|c|c|}
\hline & & \multicolumn{7}{|c|}{ case } \\
weights & $\mathrm{A}$ & $\mathrm{B}$ & $\mathrm{C}$ & $\mathrm{D}$ & $\mathrm{E}$ & $\mathrm{F}$ & $\mathrm{G}$ \\
\hline$w_{P}$ & 100.0 & 100.0 & 100.0 & 100.0 & 100.0 & 10.0 & 100.0 \\
\hline$w_{Q}$ & $\infty$ & $\infty$ & $\infty$ & $\infty$ & $\infty$ & $\infty$ & 0.0 \\
\hline$w_{s}$ & 0.1 & 10.0 & 10.0 & 10.0 & 0.1 & 10.0 & 10.0 \\
\hline$w_{t}$ & 0.1 & 0.1 & 0.1 & 10.0 & 10.0 & 10.0 & 0.1 \\
\hline$w_{p}$ & 0.1 & 0.1 & 10.0 & 0.1 & 10.0 & 10.0 & 0.1 \\
\hline
\end{tabular}

the constants appearing in these constraints being properly chosen as explained in [27].

Observe that for a connected line (i.e. $s_{i j}=1$ ) constraints (40)-(41) recover original active/reactive power flow thermal limits, (42) and (43) take the same form as (39) and (36), and (44)-(45) provides the same outcome as (26)-(27) i.e. $W_{i j}=$ $W_{j i}$ and $T_{i j}=-T_{j i}$. Otherwise, if a line is disconnected (i.e. $\left.s_{i j}=0\right)$ implies $P_{i j}=P_{j i}=0, Q_{i j}=Q_{j i}=0$ while the other constraints (42)-(45) are relaxed.

6) Relaxed voltage magnitude limits:

$$
V_{i \min }^{2}-\delta_{1 i} \leq U_{i} \leq V_{i \max }^{2}+\delta_{2 i}
$$

\section{Remarks}

A salient feature of this optimization model is that all constraints are linear except the following quadratic constraints (35), (36), (37), (38), and (43), the original MINLP problem being transformed into a simpler equivalent MIQC.

\section{NUMERICAL RESULTS}

\section{A. General details}

The MINLP-RC and MIQCP optimization models have been developed in GAMS version 23.9.3 [40] and are solved using the simple branch and bound (SBB) solver. All tests have been performed on a PC of $2.8-\mathrm{GHz}$ and $4-\mathrm{Gb}$ RAM.

We solve these optimization problems for several DSs using 7 cases (A to G, see Table I) which differ by the weights assigned to control actions allowing thereby to illustrate the approaches for various priorities given to control actions.

We choose a quadratic function for MW curtailment of DG in objective (1) for illustrative purposes and as it distributes in a fairer manner the effort of congestion removal between responsible DG units.

In order to test the optimization engine in tougher conditions constraints (20)-(22) are relaxed unless otherwise specified, the limitation of the number of control actions being implicitly handled by means of their weights assigned in the objective.

\section{B. Results on the modified 34-bus system}

1) Description of the system and optimization problem: We first illustrate the proposed approach for a $12.66 \mathrm{kV}$ benchmark distribution grid [39], which we modify by adding the following equipment:

- 6 identical DG units (G1 to G6) connected to this grid together with the equivalent generator G0 at the HV side of the substation. Table II provides the DG units 


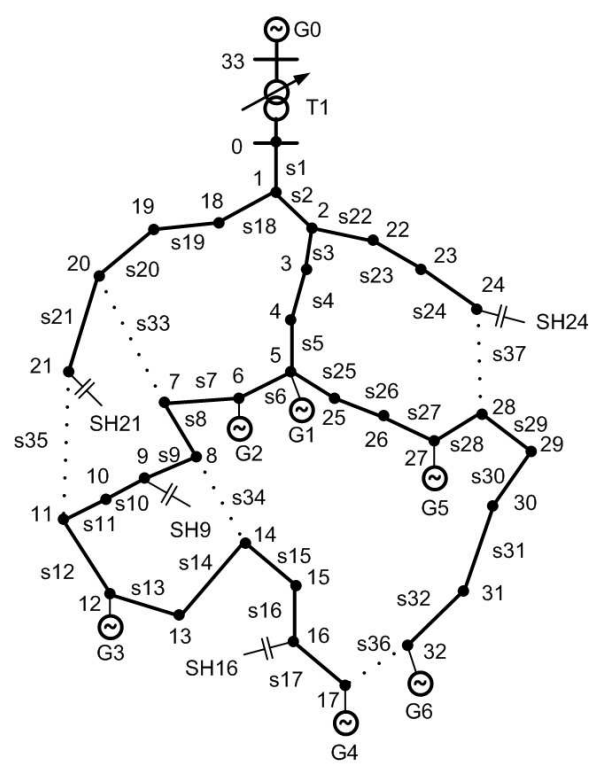

Fig. 3. Modified distribution grid

TABLE II

GENERATORS ACTIVE/REACTIVE POWERS (MW/MVAR) AND LIMITS

\begin{tabular}{|c|c|c|c|c|c|c|}
\hline gen & $P_{g}^{\mathrm{O}}$ & $Q_{g}^{\mathrm{O}}$ & $P_{g}^{\mathrm{min}}$ & $P_{g}^{\max }$ & $Q_{g}^{\mathrm{min}}$ & $Q_{g}^{\max }$ \\
\hline \hline G0 & -2.04 & 1.5 & -5.0 & 10.0 & -2.0 & 8.0 \\
\hline G1 & 1.0 & 0.0 & 0.1 & 1.0 & -0.2 & 0.1 \\
\hline G2 & 1.0 & 0.0 & 0.1 & 1.0 & -0.2 & 0.1 \\
\hline G3 & 1.0 & 0.0 & 0.1 & 1.0 & -0.2 & 0.1 \\
\hline G4 & 1.0 & 0.0 & 0.1 & 1.0 & -0.2 & 0.1 \\
\hline G5 & 1.0 & 0.0 & 0.1 & 1.0 & -0.2 & 0.1 \\
\hline G6 & 1.0 & 0.0 & 0.1 & 1.0 & -0.2 & 0.1 \\
\hline
\end{tabular}

active/reactive powers at the base case and their physical limits.

- 4 identical shunt capacitors (SH9, SH16, SH21, and SH24) located at nodes 9, 16, 21, 24.

- one HV/MV LTC transformer at the substation.

The other characteristics of this test system are: 34 buses, 37 lines, 32 sectionalizing switches, and 5 tie switches. The overall load in the base case is $3715 \mathrm{~kW}$ and $2300 \mathrm{kVar}$.

Figure 3 shows the one-line diagram of this network.

We assume voltages limits of $0.95 / 1.05$ p.u. at all nodes.

The complete set of control variables in the optimization problem is made of:

- the active/reactive powers of the 6 DG units (together with their status on/off) and of the equivalent generator G0. Note that the set of simulations A to F assume the DG units operate under unitary power factor (i.e. no reactive power is produced/absorbed), while in case $\mathrm{G}$ they are allowed to reschedule freely reactive power between bounds.

- 9 remotely controlled switches (all 5 tie switches and the following sectionalizing switches: s7, s20, s23, and s27).

- the tap of the LTC transformer at substation, for which we consider $\left|t_{a p_{33} 0}\right|=25$ discrete tap positions with a ratio step variation $\Delta r_{330}=0.01 \mathrm{pu}$, and that $t_{330}^{0}=14$.

- the step position of the 4 identical shunt capacitors. Each shunt has $\left|s h_{i}\right|=8$ discrete positions (ranging from 1 to 8 ), is initially on position $p_{s h i}=2$, and the reactive power per step is $\Delta b_{s h i} V_{i}^{2}=0.3$ MVar.

As a consequence, neglecting the optimization of continuous variables, the complete combinatorial space of discrete variables is very large $2^{6} \times 2^{9} \times 25^{1} \times 8^{4}=3,355,443,200$ precluding an enumeration approach and fully justifying the use of optimization approaches.

2) Comparison of optimization models for overvoltage constraints alleviation: Table III reports the results obtained with both models for the problem of voltage raise removal. If the cost of discrete controls switching is expensive, as in case E, the whole effort of voltage congestion removal is undertaken by the DG MW curtailment which leads to the largest amount of curtailment among all scenarios (1.26 MW is curtailed out of the total of $6.0 \mathrm{MW}$ ). As the cost of discrete controls switching decreases, as in other 6 cases, the amount of DG curtailment reduces significantly or is not necessary.

Notice that in some cases the voltage constraints are removed without curtailing DG. Actually, if the voltage constraints can be removed without DG curtailment the objective (1) minimizes the total (weighted) number of switching actions on switches, LTCs and shunts. This explains why in case A the problem is solved by three switching actions (i.e. closing the tie switch s35, opening the sectionalizing switch s7, and reducing the reactive power injection of shunt SH16) whereas in case $\mathbf{B}$, as the cost of network reconfiguration $w_{s}$ gets higher, 5 switching actions are needed (three on the tap position and two on shunts SH9 and SH16). In case C, as the cost of grid reconfiguration and shunt switchings increase sole the LTC tap cannot remove the congestion and some MW curtailment on DG is required. In case D, as the cost of network reconfiguration and LTC tap are high whereas the shunt switching is cheap, MW curtailment of DG is again needed. Finally, in case F, where one assumes that each DG can reschedule at no cost reactive power between the bounds, expectedly at the optimum all DG units absorb reactive power reaching the minimal limit of $-0.2 \mathrm{MVar}$; these actions together with the decrease of shunt SH16 MVar injection save the MW curtailment of DG.

Both optimization models converge to the same solution in all cases, except case E, where the control actions provided by the model MIQC slightly differ (i.e. it uses switch s35 instead of s37), the latter leading to a better value of the objective ( 0.2 vs. 0.6). The computational effort of both models is small and the MINLP-RC model is faster in all cases.

\section{Results on the 137-bus system}

We now consider a 137-bus $13.8 \mathrm{kV}$ real-life distribution system in Brazil [41] that we modify by adding the following equipment: one LTC transformer with 25 discrete tap positions, 6 identical shunt capacitors with 8 discrete positions, and 18 identical DG units each injecting 1 MW/0.3 MVar in the base case and operating under 0.95 constant power factor. These control means have the same characteristics as for the 34-bus system. We furthermore consider that 29 switches have remote control capability (all 21 tie switches and 8 sectionalizing switches i.e. one for each feeder). 
TABLE III

34-BUS SYSTEM: CONTROLS CHANGE, OBJECTIVE, AND CPU TIMES

\begin{tabular}{|c|c|c|c|c|c|c|c|}
\hline \multirow[b]{2}{*}{ controls } & \multicolumn{7}{|c|}{ case } \\
\hline & A & B & $\mathrm{C}$ & D & E & $\mathrm{F}$ & $\mathrm{G}$ \\
\hline \multicolumn{8}{|c|}{ model MINLP-RC } \\
\hline G1 & $\overline{0.0}$ & 0.0 & -0.05 & -0.04 & $\overline{0.0}$ & \begin{tabular}{c|c|c|}
-0.10 \\
\end{tabular} & 0.0 \\
\hline $\mathrm{G} 2$ & $\overline{0.0}$ & 0.0 & -0.05 & -0.04 & $\overline{0.0}$ & -0.10 & 0.0 \\
\hline G3 & 0.0 & 0.0 & -0.17 & -0.13 & 0.0 & -0.36 & 0.0 \\
\hline G4 & 0.0 & 0.0 & -0.25 & -0.19 & 0.0 & -0.52 & 0.0 \\
\hline G5 & 0.0 & 0.0 & -0.05 & -0.03 & 0.0 & -0.09 & 0.0 \\
\hline G6 & 0.0 & 0.0 & -0.04 & -0.03 & 0.0 & $\begin{array}{ll}-0.09 \\
\end{array}$ & 0.0 \\
\hline switches & s7, s35 & & & & $\begin{array}{c}\mathrm{s} 7, \mathrm{~s} 33 \\
\mathrm{~s} 23, \mathrm{~s} 36 \\
\mathrm{~s} 27, \mathrm{~s} 37\end{array}$ & & \\
\hline T1 & & -3 & -3 & & & & \\
\hline SH9 & & -1 & & -1 & & & \\
\hline SH16 & -1 & -1 & & -1 & & & -1 \\
\hline SH21 & & & & -1 & & & \\
\hline SH24 & & & & -1 & & & \\
\hline objective (p.u.) & 0.3 & 0.5 & 10.635 & 6.471 & 0.6 & 4.372 & 0.1 \\
\hline curtailed MW & 0.0 & 0.0 & 0.61 & 0.46 & 0.0 & 1.26 & 0.0 \\
\hline switchings & 3 & 5 & 3 & 4 & 6 & 0 & 1 \\
\hline time $(\mathrm{s})$ & 1.69 & 0.39 & 0.60 & 0.60 & 3.97 & 0.43 & 0.56 \\
\hline \multicolumn{8}{|c|}{ model MIQC } \\
\hline time $(\mathrm{s})$ & 3.68 & 0.90 & 0.98 & 0.78 & 9.24 & 0.70 & 0.60 \\
\hline
\end{tabular}

TABLE IV

137-BUS SYSTEM: CONTROLS CHANGE, OBJECTIVE, AND CPU TIMES

\begin{tabular}{|c|c|c|c|c|c|c|c|}
\hline \multirow[b]{2}{*}{ controls } & \multicolumn{7}{|c|}{ case } \\
\hline & A & B & $\mathrm{C}$ & D & $\mathrm{E}$ & $\mathrm{F}$ & G \\
\hline \multicolumn{8}{|c|}{ model MINLP-RC } \\
\hline$\overline{\overline{\mathrm{G} 1}}$ & $\overline{0.0 .0}$ & 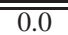 & 0.0 & $\overline{0.0}$ & $\overline{\overline{0.0}}$ & $\overline{-0.05}$ & $\overline{0.0}$ \\
\hline$\overline{\mathrm{G}} 2$ & 0.0 & 0.0 & 0.0 & 0.0 & 0.0 & -0.08 & 0.0 \\
\hline G3 & 0.0 & 0.0 & 0.0 & 0.0 & 0.0 & -0.11 & 0.0 \\
\hline$\overline{\mathrm{G} 4}$ & 0.0 & 0.0 & 0.0 & 0.0 & 0.0 & -0.12 & 0.0 \\
\hline G5 & 0.0 & 0.0 & 0.0 & 0.0 & 0.0 & -0.14 & 0.0 \\
\hline G6 & 0.0 & 0.0 & 0.0 & 0.0 & 0.0 & -1.00 & 0.0 \\
\hline G7 & 0.0 & 0.0 & 0.0 & 0.0 & 0.0 & -0.22 & 0.0 \\
\hline G8 & 0.0 & 0.0 & 0.0 & 0.0 & 0.0 & -0.15 & $\overline{0.0}$ \\
\hline switches & & & & & s81,s138 & & \\
\hline$\overline{\mathrm{T} 1}$ & $\overline{-2}$ & $\overline{-2}$ & $\overline{-2}$ & -2 & $\overline{-1}$ & & \\
\hline objective (p.u.) & 0.2 & 0.2 & 0.2 & 20.0 & 10.2 & 11.03 & $\overline{0.0}$ \\
\hline curtailed MW & 0.0 & 0.0 & 0.0 & 0.0 & 0.0 & 1.87 & 0.0 \\
\hline switchings & 2 & 2 & 2 & 2 & 3 & 0 & 0 \\
\hline time $(\mathrm{s})$ & 3.67 & 1.66 & 1.65 & 2.97 & $5.87 \star$ & 2.64 & 0.12 \\
\hline \multicolumn{8}{|c|}{ model MIQC } \\
\hline objective (p.u.) & $\overline{0.3}$ & 0.2 & 0.2 & 20.0 & $\overline{10.3}$ & 11.03 & $\overline{0.0}$ \\
\hline curtailed MW & 0.0 & 0.0 & 0.0 & 0.0 & 0.0 & 1.87 & 0.0 \\
\hline switchings & 3 & 2 & 2 & 2 & 4 & 0 & 0 \\
\hline time $(\mathrm{s})$ & 108.7 & 10.2 & 10.3 & 8.96 & $22.79 \star$ & 105.8 & 0.15 \\
\hline \multicolumn{8}{|c|}{ model MINLP-RC - all switches fixed } \\
\hline time (s) & 1.29 & 1.28 & 1.29 & 1.63 & 1.24 & 1.45 & 0.10 \\
\hline \multicolumn{8}{|c|}{ model MIQC - all switches fixed } \\
\hline time (s) & 2.84 & 2.80 & 2.92 & 3.92 & 3.94 & 3.52 & 0.13 \\
\hline
\end{tabular}

Table IV provides the results obtained with both models for the problem of voltage raise removal in 7 cases.

We notice that in these cases the MINLP-RC model converges very fast and outperforms the MIQC model in terms of both objective value and especially computational speed. However, in case E, both models reach the maximum time execution constraint which was set to 120 seconds. Actually in this case the solver cannot find a sub-optimal solution of acceptable quality in the allotted time, although a usable
TABLE V

CHARACTERISTICS OF TEST SYSTEMS

\begin{tabular}{|c|c|c|c|c|c|c|}
\hline & \multicolumn{6}{|c|}{ number of: } \\
name & nodes & lines & LTCs & shunts & DG units & RCS \\
\hline D0 & 137 & 156 & 1 & 6 & 18 & 29 \\
\hline D1 & 273 & 312 & 2 & 12 & 36 & 29 \\
\hline D2 & 409 & 468 & 3 & 18 & 54 & 29 \\
\hline D3 & 545 & 624 & 4 & 24 & 72 & 29 \\
\hline D7 & 1089 & 1248 & 8 & 48 & 144 & 29 \\
\hline
\end{tabular}

solution is found quickly and it does not change even after running the program for longer time. Such cases can be mitigated by further imposing a reasonable limit on the number of switches status change (20) which reduces dramatically the size of the combinatorial space. Thus, the CPU times reported in Table IV with both models in case $\mathrm{E}$ have been obtained with the number of status change of switches being limited to 2 (i.e. a swap between an open and a closed switch).

We also noticed that in case F generator G6 is completely shut down, which proves that the binary statuses of DG units are properly handled.

We furthermore investigate the impact on the computational time of not using remotely controlled switches as control variables and report the obtained results in the same table. We notice that both models converge very fast as the remaining discrete variables are more easily manageable by continuous relaxation. We therefore conclude that as expected the major computational effort is due to inclusion of switches in the optimization.

The computational times are generally slightly larger than for the 34-bus system, especially due to the larger combinatorial space when switches are considered in optimization.

\section{Experiments on larger systems}

We further analyze how the proposed approaches scale with the problem size. Specifically, we consider distribution systems of increasing size obtained by duplicating the 137-bus system of sub-section IV-C while keeping as common node the high voltage side of the HV/MV LTC transformer.

Table $\mathrm{V}$ provides the main characteristics of the test systems, where the acronym "DY" indicates how many times the original system has been duplicated (e.g. D0 corresponds to the original system). Note that only the number of RCS has not been duplicated, as the assumed number is already larger than the level of automation of current DSs or future active DSs. Furthermore, the numbers of shunts and DG units have been chosen larger than in reality in order to test the solvers performances.

We first run the MINLP-RC model for the 7 different cases on the largest grid model and report the obtained computational times in the row labeled D7-full in Table VI, where the maximum running time constraint has been arbitrarily set to 600 seconds. One can observe that as expected in some cases (D, E, and F) a sub-optimal solution of acceptable quality (i.e. that meets the solver default tolerance for the optimality gap) has not been obtained in the allotted running time. Note however, that a feasible discrete solution is found in all cases 
TABLE VI

CPU TIMES ( $\mathrm{S}$ )

\begin{tabular}{|c|c|c|c|c|c|c|c|}
\hline & & case \\
system & $\mathrm{A}$ & $\mathrm{B}$ & $\mathrm{C}$ & $\mathrm{D}$ & $\mathrm{E}$ & $\mathrm{F}$ & $\mathrm{G}$ \\
\hline \hline \multicolumn{8}{|c|}{ model MINLP-RC } \\
\hline \hline D0 & 4.09 & 1.86 & 1.78 & 2.85 & 5.87 & 2.18 & 0.12 \\
\hline D1 & 3.54 & 2.67 & 2.59 & 35.73 & 32.75 & 67.89 & 0.25 \\
\hline D2 & 9.01 & 13.84 & 9.16 & 23.47 & 109.32 & $5.16^{*}$ & 0.41 \\
\hline D3 & 12.30 & 16.97 & 17.95 & 44.09 & $57.33^{*}$ & $8.43^{*}$ & 0.63 \\
\hline D7 & $53.41^{*}$ & $5.49^{*}$ & $4.98^{*}$ & $22.43^{*}$ & $103.14^{*}$ & $10.27^{*}$ & 1.69 \\
\hline D7-full & 39.67 & 38.87 & 35.29 & 600.0 & 600.0 & 600.0 & 1.91 \\
\hline \hline \multicolumn{8}{|c|}{ model MIQC } \\
\hline \hline D0 & 6.57 & 8.84 & 8.97 & 12.83 & 22.28 & 89.98 & 0.15 \\
\hline D1 & 34.71 & 55.19 & 26.4 & 50.37 & failed & 343.1 & 2.85 \\
\hline D2 & $600.0^{*}$ & 87.18 & 87.17 & $600.0^{*}$ & $600.0^{*}$ & $600.0^{*}$ & 9.60 \\
\hline
\end{tabular}

in few tens of seconds and hence even if the solver needs to be prematurely stopped it will provide a usable solution.

As slow convergence of the proposed optimization models is sometimes to be expected as the number of discrete variables and the system size increase significantly we propose hereafter further reasonable problem relaxation to reduce the huge combinatorial space and produce solutions usable:

- the number of switching actions on remotely controlled switches has been limited to 2 in (20);

- in some cases denoted with an asterisk * the binary variables modeling DG connection status are relaxed by assuming that none DG unit is completely shut down, i.e. $s_{g i}=1, i \in G$ in (2).

Under these additional assumptions Table VI provides the computational times obtained with the two optimization models MINLP-RC and MIQC on the various test systems. These results clearly show that the MINLP-RC model is consistently faster than the MIQC. The simulations with the latter model have not been pursued as it does not perform satisfactorily for small and medium size systems.

Note that under these assumed problem relaxations the MINLP-RC model scales acceptably to large scale problems.

\section{CONCLUSIONS AND FUTURE WORKS}

This paper has proposed a centralized optimization approach for the voltage constraints management (VCM) in active distribution systems. A salient feature of the approach is its comprehensive and accurate modeling of the coordinated interaction between control means (DG units connection status and active/reactive powers, remotely controlled switches (RCS), LTC ratio, and shunt banks). We have proven the interest and feasibility of the approach on a snapshot basis for various distribution systems up to 1089 buses.

An important finding of this work is that the use of RCS for VCM leads in some cases to a significant reduction of MW curtailed, RCS appearing thereby as an effective means to accommodate larger amounts of DG in distribution grids. The proposed approach can also serve for assessing the gain in terms of larger DG penetration level due to the use of RCS.

The results show that although the MIQC model is mathematically more appealing the MINLP-RC model is faster and scales better with the problem size. This demonstrates that modeling switching actions by introducing a more than quadratic yet mild nonlinearity as in MINLP-RC model (see constraints (13)-(14)) is more effective than enforcing active and reactive power flows at both sides of open branches to be zero as in the MIQC model (see constraints (40)(41)). Furthermore, the MINLP-RC is more generic allowing application to meshed distribution systems whereas MIQC applies only to radial DSs.

Extensive numerical results allow concluding that the MINLP-RC model converges generally fast for a reasonable range of distribution system sizes and number of discrete variables and is therefore a candidate for VCM in active DSs. However, as the system size and number of discrete variables increase significantly, in order to reduce the computational burden and obtain solutions acceptably fast, some ambitious modeling options should be relaxed in a reasonable way (e.g. limiting the number of switching actions on RCS and not modeling the complete shut down of DG units).

Significant improvement of the obtained computational times is expected thanks to:

- further progress and parallelization of MINLP algorithms;

- use of appropriate computer architecture;

- assessment of switching actions by DSO at (operational) planning stage [9], [38], ending up with a practical limited set of potential switching pairs to be checked on-line as voltage constraints occur. Such a procedure would greatly simplify our optimization models allowing problem decomposition, further enabling parallel processing, and focusing on discrete variables for which continuous relaxation works acceptably.

- grid model reduction using well-known generic network equivalents (e.g. Ward, Dimo) [43] or techniques specific for distribution systems [44].

In order to potentially overcome the limitations of the MINLP-RC model for larger distribution systems and larger sets of discrete variables, few alternative optimization approaches, that have been primarily devised for different purposes, can also be adapted to VCM problem. These can be classified in two categories: direct and decomposition methods. The former class includes accurate mixed integer (convex) conic programming (MICP) approach [34], approximate MICP [45], and reasonable MILP approximations [8], [9], [34], [35]. The latter class decomposes the discrete variable set into two subsets [46]: variables with highly nonlinear impact on the system (e.g. comprising binary variables such as switches/breakers status and DG connection status) and discrete variables with comparatively less nonlinear behavior (e.g. LTC ratio and shunt banks) so as to enable using suitable solution techniques for each subset of variables (e.g. MILP for the former [8], [34], [35] and heuristic techniques relying on probabilistic progressive round-off [24] or sensitivities and merit functions [47] for the latter).

Future work is planned to:

- assess the approach in the context of closed-loop on-line application as well as in terms of energy savings and capacity factor by using time-series [6], [15];

- extend the optimization model by imposing additional 
constraints on fault current levels [42] so as to preserve the feeders' protection selectivity when transferring DG units from one feeder to another;

- develop the optimization method so as to consider unbalanced operating conditions [48];

- extend the approach for other DSO needs (e.g. minimization of losses) whenever the system operates in a normal state.

\section{ACKNOWLEDGMENTS}

This work is conducted as part of the "Reliable and Efficient Distributed Electricity Generation in Smart Grids" (REDESG) project within the "CREOS-SnT Framework Program". F. Capitanescu and I. Bilibin are supported by the Fonds National de la Recherche, Luxembourg (C11/SR/1278568).

E. Romero Ramos acknowledges P09-TEP-5170 project financed by Junta de Andalucia.

\section{REFERENCES}

[1] J.J. Graniger and S. Civanlar, "Volt/Var Control on Distribution Systems with Lateral Branches Using Shunt Capacitors and Voltage Regulators Part I: The Overall Problem", IEEE Trans. on PAS, vol. PAS-104, no. 11, pp. 3278-3282, 1985 .

[2] I. Roytelman, B.K. Wee, R.L. Lugtu, "Volt/var control algorithm for modern distribution management system", IEEE Trans. on Power Syst., vol. 10, no. 3, pp. 1454-1460, 1995.

[3] A. Keane, L.F. Ochoa, C.L.T. Borges, G.W. Ault, A. D. AlarconRodriguez, R.A.F. Currie, F. Pilo, C. Dent, and G.P. Harrison, "Stateof-the-Art Techniques and Challenges Ahead for Distributed Generation Planning and Optimization”, IEEE Trans. on Power Syst., vol. 28, no. 2, pp. 1493-1502, 2013.

[4] N. Markushevich, "The benefits and challenges of the integrated volt/var optimization in the smart grid environment", IEEE PES General Meeting, Detroit, USA, 2011.

[5] S. N. Liew and G. Strbac, "Maximising penetration of wind generation in existing distribution networks", IEE Proc., Gen., Transm. \& Distrib., vol. 149, pp. 256-262, 2002.

[6] T. Boehme, G.P. Harrison, and A.R. Wallace, "Assessment of distribution network limits for non-firm connection of renewable generation", IET Renewable Power Generation, vol. 4, pp. 64-74, 2010.

[7] Q. Zhou and J. Bialek, "Generation curtailment to manage voltage constraints in distribution networks", IET Gen., Transm. \& Distrib., vol. 1, pp. 492-498, 2007.

[8] A. Borghetti, "Using mixed integer programming for the volt/var optimization in distribution feeders", Electric Power Systems Research, vol. 98, no. 8, pp 39-50, 2013.

[9] F. Pilo, G. Pisano, and G. Soma, "Optimal Coordination of Energy Resources With a Two-Stage Online Active Management", IEEE Trans. on Industrial Electronics, vol. 58, no. 10, pp. 4526-4537, 2011.

[10] G. Valverde and T. Van Cutsem, "Model predictive control of voltages in active distribution networks", IEEE Trans. Smart Grid, in press.

[11] A.A. Aquino-Lugo, R. Klump, and T.J. Overbye, "A Control Framework for the Smart Grid for Voltage Support Using Agent-Based Technologies", IEEE Trans. Smart Grid, vol. 2, no. 1, pp. 173-180, 2011.

[12] S. Deshmukh, B. Natarajan, and A. Pahwa, "Voltage/VAR Control in Distribution Networks via Reactive Power Injection Through Distributed Generators", IEEE Trans. Smart Grid, in press.

[13] P. N. Vovos, A.E. Kiprakis, A.R. Wallace and G.P. Harrison, "Centralized and Distributed Voltage Control: Impact on Distributed Generation Penetration", IEEE Transactions on Power Syst., vol. 22, no. 1, pp. 476-483, 2007.

[14] P.M.S. Carvalho, P.F. Correia, and L.A.F.M. Ferreira, "Distributed Reactive Power Generation Control for Voltage Rise Mitigation in Distribution Networks", IEEE Trans. on Power Syst., vol. 23, no. 2 , pp. 766-772, 2008.

[15] T. Sansawatt, L.F. Ochoa, and G.P. Harrison, "Smart decentralized control of DG for voltage and thermal constraint management", IEEE Trans. on Power Syst., vol. 27, no. 3, pp. 1637-1645, 2012.
[16] M. Fila, D. Reid, G.A. Taylor, P. Lang, and M.R. Irving, "Coordinated voltage control for active network management of distributed generation", IEEE Power \& Energy Society General Meeting, 2009.

[17] M. E. Baran and I. M. El-Markabi, "A multiagent-based dispatching scheme for distributed generators for voltage support on distribution feeders", IEEE Trans. Power Syst., vol. 22, no. 1, pp. 52-59, 2007.

[18] M. Brenna, E. De Berardinis, L. Delli Carpini, F. Foiadelli, P. Paulon, P. Petroni, G. Sapienza, G. Scrosati, and D. Zaninelli, "Automatic Distributed Voltage Control Algorithm in Smart Grids Applications", IEEE Trans. Smart Grid, in press.

[19] H. E. Farag, E. F. El-Saadany, and R. Seethapathy, "A two ways communication-based distributed control for voltage regulation in smart distribution feeders”, IEEE Trans. Smart Grid, vol. 3, no. 1, pp. 271-281, 2012.

[20] Y. Hen-Geul, D.F. Gayme, and S.H. Low, "Adaptive VAR Control for Distribution Circuits With Photovoltaic Generators", IEEE Trans. on Power Syst., vol.27, no.3, pp. 1656-1663, 2012.

[21] F. A. Viawan and D. Karlsson, "Combined local and remote voltage and reactive power control in the presence of induction machine distributed generation," IEEE Trans. Power Syst., vol. 22, no. 4, pp. 2003-2012, 2007.

[22] P.M.S. Carvalho, L.A.F.M. Ferreira, and A.J.C. da Silva, "A decomposition approach to optimal remote controlled switch allocation in distribution systems", IEEE Trans. on Power Syst., vol. 20, no. 2, pp. 1031-1036, 2005

[23] M. Lavorato, J.F. Franco, M.J. Rider, and R.Romero, "Imposing Radiality Constraints in Distribution System Optimization Problems", IEEE Trans. on Power Syst., vol. 27, no. 1, pp. 172-180, 2012.

[24] P.J. Macfie, G.A. Taylor, M.R. Irving, P. Hurlock, and Hai-Bin Wan, "Proposed Shunt Rounding Technique for Large-Scale Security Constrained Loss Minimization," IEEE Trans. on Power Syst., vol. 25, no. 3, pp. 1478-1485, 2010.

[25] L. Platbrood, S. Fliscounakis, F. Capitanescu, P. Panciatici, C. Merckx, and M. Ortega-Vazquez, "Deliverable D3.2: development of prototype software for system steady-state optimization of the European transmission system", PEGASE project, available on-line at http://www.fp7pegase.com, 2011.

[26] A. Gomez Exposito and E. Romero Ramos, "Reliable load flow technique for radial distribution networks", IEEE Trans. on Power Syst., vol. 14, no. 3, August 1999, pp. 1063-1069.

[27] E. Romero Ramos, J. Riquelme Santos, and J. Reyes, "A simpler and exact mathematical model for the computation of the minimal power losses tree", Electric Power Systems Research, vol. 80, no. 5, pp. 562571,2010

[28] I. Bilibin, F. Capitanescu, J. Sachau, "Overloads Management in Active Radial Distribution Systems: an Optimization Approach Including Network Switching", IEEE Powertech conference, Grenoble (France), June 2013.

[29] Per Hallberg (Chair) et al., "Active Distribution System Management a key tool for the smooth integration of distributed generation", Eurelectric TF Active System Management, 2013, available on-line.

[30] M.J. Dolan, E.M. Davidson, I. Kockar, G.W. Ault, and S.D.J. McArthur, "Distribution power flow management utilizing an online optimal power flow technique", IEEE Trans. on Power Syst., vol. 27, no. 2, pp. 790-799, May 2012.

[31] J.M. Lopez-Lezama, A. Padilha-Feltrin, J. Contreras, and J.I. Munoz, "Optimal Contract Pricing of Distributed Generation in Distribution Networks", IEEE Trans. on Power Syst., vol. 26, no. 1, pp.128-136, 2011.

[32] A.C. Rueda-Medina and A. Padilha-Feltrin, "Distributed Generators as Providers of Reactive Power Support - A Market Approach" IEEE Trans. on Power Syst., in press.

[33] P. Cuffe, P. Smith, and A. Keane, "Capability Chart for Distributed Reactive Power Resources", IEEE Trans. on Power Systems, to appear 2013.

[34] R. Jabr, R. Singh, and B.C. Pal, "Minimum Loss Network Reconfiguration Using Mixed-Integer Convex Programming" IEEE Trans. on Power Systems, vol. 27, no. 2, 2012, pp. 1106-1115.

[35] J.F. Franco, M.J. Rider, M. Lavorato, R. Romero, "A mixed-integer LP model for the reconfiguration of radial electric distribution systems considering distributed generation", Electric Power Systems Research, Vol. 97, no. 4, 2013, pp. 51-60.

[36] R. Singh, B.C. Pal, R.A. Jabr, and R.B. Vinter, "Meter placement for distribution system state estimation: an ordinal optimization approach", IEEE Trans. on Power Systems, vol. 26, no. 4, pp. 2328-2335, 2011. 
[37] N. Nusrat, M. Irving, and G. Taylor, "Novel meter placement algorithm for enhanced accuracy of distribution system state estimation" IEEE PES General Meeting, San Diego (USA), July 2012.

[38] L.L. Pfitscher, D.P. Bernardon, L.N. Canha, V.F. Montagner, V.J. Garcia, A.R. Abaide, "Intelligent system for automatic reconfiguration of distribution network in real time", Electric Power Systems Research, Vol. 97, No. 4, 2013, pp. 84-92.

[39] M.E. Baran and F.F. Wu, "Network Reconfiguration in Distribution Systems for Loss Reduction and Load Balancing", IEEE Trans. Power Delivery, vol. 4, no. 2, 1989, pp. 1401-1497.

[40] B.A. McCarl, "GAMS User Guide", Version 23.8, 2012. Available online: www.gams.com.

[41] J.R.S. Mantovani, F. Casari, and R. A. Romero, "Reconfiguracao de sistemas de distribuicao radiais utilizando o criterio de queda de tensao", SBA Controle \& Automacao, vol. 11, no. 3, pp. 150-159, 2000.

[42] P. Vovos, J. Bialek, "Direct incorporation of fault level constraints in optimal power flow as a tool for network capacity analysis", IEEE Trans. on Power Syst., vol. 20, no. 4, pp. 2125-2134, 2005.

[43] A. Akhavein, M. Fotuhi Firuzabad, R. Billinton and D. Farokhzad, "Review of reduction techniques in the determination of composite system adequacy equivalents", Electric Power Systems Research, vol. 80, no. 12, pp. 1385-1393, 2010.

[44] J. Liu, B. Pengxiang, Z. Yanqing, and W. Xiaomeng, "Power Flow Analysis on Simplified Feeder Modeling", IEEE Transactions on Power Delivery, vol. 19, no. 1, pp. 279-287, 2004.

[45] J.A. Taylor and F.S. Hover, "Convex Models of Distribution System Reconfiguration” IEEE Trans. on Power Systems, vol. 27, no. 3, 2012, pp. 1407-1413.

[46] F. Capitanescu, J.L. Martinez Ramos, P. Panciatici, D. Kirschen, A. Marano Marcolini, L. Platbrood, and L. Wehenkel. "State-of-the-art, challenges, and future trends in security-constrained optimal power flow", Electric Power System Research, vol. 81, no. 8, pp 1731-1741, 2011.

[47] F. Capitanescu and L. Wehenkel. "Sensitivity-based approaches for handling discrete variables in optimal power flow computations", IEEE Trans. on Pow. Syst., vol. 25, no. 4, pp. 1780-1789, 2010.

[48] S. Bruno, S. Lamonaca, G. Rotondo, U. Stecchi and M. La Scala, "Unbalanced Three-Phase Optimal Power Flow for Smart Grids", IEEE Transactions on Industrial Electronics, vol. 58, no. 10, pp. 4504-4513, 2011.

Florin Capitanescu graduated in Electrical Power Engineering from the University "Politehnica" of Bucharest in 1997. He obtained the Ph.D. degree from the University of Liège in 2003. His main research interests include the application of optimization methods in the field of planning, operation, and control of transmission and distribution systems.

Ilya Bilibin graduated in applied mathematics and physics from the Moscow Institute of Physics and Technology in 2009. His main research interests are power system adequacy and security, distributed generation integration, electricity market modeling and optimization methods. He is currently a Ph.D. student with SnT, University of Luxembourg.

Esther Romero Ramos was born in Spain, in 1967. She graduated in electrical engineering at University of Sevilla in 1992, where she obtained the Ph.D. degree in 1999. From 1992 to 1993 she worked for Sainco. Since 1993 she has been with the Department of Electrical Engineering, University of Sevilla, where she is currently an Associate Professor. She is interested in State Estimation, Load Flow problems, Optimal Power System Operation and Analysis, and Control of Distribution Systems. 